\title{
SPIN TRACKING AT THE INTERNATIONAL LINEAR COLLIDER *
}

\author{
I.R. Bailey ${ }^{1,2, \dagger}$, D.P. Barber ${ }^{1,3}$, A. Hartin ${ }^{4}$, L.I. Malysheva ${ }^{1,2}$, G.A. Moortgat-Pick ${ }^{1,5}$, \\ E. Baynham ${ }^{7}$, A. Birch ${ }^{1,6}$, T. Bradshaw ${ }^{7}$, A. Brummitt ${ }^{7}$, S. Carr ${ }^{7}$, J.A. Clarke ${ }^{1,6}$, \\ P. Cooke ${ }^{2}$, J.B. Dainton ${ }^{1,2}$, Y. Ivanyushenkov ${ }^{7}$, L.J. Jenner ${ }^{1,2}$, A. Lintern ${ }^{7}$, O.B. Malyshev ${ }^{1,6}$, \\ J. Rochford ${ }^{7}$, D.J. Scott ${ }^{1,2,6}$, L. Zang ${ }^{1,2}$ \\ ${ }^{1}$ Cockcroft Institute, Daresbury Laboratory, Warrington, Cheshire WA4 4AD, U.K. \\ 2 Department of Physics, University of Liverpool, Oxford St., Liverpool, L69 7ZE, U.K. \\ ${ }^{3}$ DESY, Deutsches Elektronen Synchrotron, Notkestraße 85, D-22607 Hamburg, Germany \\ ${ }^{4}$ John Adams Institute, Oxford University Physics, Oxford OX1 3RH, U.K. \\ ${ }^{5}$ Institute of Particle Physics Phenomenology, University of Durham, Durham DH1 3LE, U.K. \\ ${ }^{6}$ STFC ASTeC Daresbury Laboratory, Daresbury, Warrington, Cheshire WA4 4AD, U.K. \\ ${ }^{7}$ STFC Rutherford Appleton Laboratory, Chilton, Didcot, Oxfordshire OX11 0QX, U.K.
}

\section{Abstract}

Polarized $e^{-}$and $e^{+}$beams are foreseen for the future International Linear Collider (ILC). High precision physics requires the polarization of both beams to be known with a relative uncertainty of about $0.1 \%$ or better. Therefore all possible depolarizing effects that could operate between the polarized sources and the interaction regions have to be under full control.

This report gives a brief summary of ongoing work on the ILC spin-dynamics concentrating on recent results for depolarizing effects in the ILC damping rings, main linac, beam delivery system and beam-beam interactions. The polarization dependence of incoherent and second order coherent background processes have been taken into account as well as the treatment of spin precession in strong fields.

\section{OVERVIEW}

The full physics potential of the ILC can be realized only by utilising polarized $e^{-}$and $e^{+}$beams [1]. Polarized $e^{-}$ with a polarization between $80 \%$ and $90 \%$ are foreseen for the baseline machine design. In the current Reference Design Report (RDR) [2] of the ILC a helical undulator based positron source [3] has been chosen as the most reliable solution for producing the required flux of order $10^{14}$ positrons per second. The design produces positrons via an electromagnetic shower instigated in a thin target by incident circularly polarized synchrotron radiation produced by the undulator operating on the main ILC $e^{-}$beam. Even in the baseline design, that foresees only an approximately $100 \mathrm{~m}$ long helical undulator, a $e^{+}$polarization of about $30 \%$ can be achieved and could be used for enhancing physics analyses. It is, however, important to ensure that no significant polarization is lost during the transport of the $e^{-}$and $e^{+}$beams from their sources to the interaction

\footnotetext{
* This work is supported in part by the Commission of the European Communities under the $6{ }^{\text {th }}$ Framework Programme "Structuring the European Research Area", contract number RIDS-011899.

$\dagger$ i.r.bailey@ cockcroft.ac.uk
}

region.

The largest depolarizing effects are expected to result from the collision of the two beams at the interaction point(s) themselves [7]. The status of our analysis of the polarization aspects of the beam-beam interactions including incoherent and second order coherent background processes is presented in the first section of this report.

Transport elements between the sources and interaction points which can also contribute to a loss of polarization include the initial acceleration structures, transport lines to the damping rings, the damping rings, the spin rotators, the main linacs, and the high energy beam delivery systems [5]. A synopsis of our studies of depolarization in the ILC damping rings, main linac and the beam delivery system is given in the second section of this report.

\section{BEAM-BEAM INTERACTIONS}

The program CAIN [6] evaluates analytically the two sources of depolarization during beam-beam interaction at the linear collider: the spin precession as well as the spinflip Sokolov-Ternov (S-T) effect. Usually the spin precession effect is dominant, but at higher energy the depolarization due to the S-T effect increases [7]. Studies and extensions of the CAIN physics model are presented below.

\section{Depolarization from Spin Precession}

Spin precession is described by the Thomas-BargmannMichel-Telegdi (T-BMT) equation:

$$
\frac{\mathrm{d} \vec{S}}{d t}=\frac{-e}{m \gamma}\left[(\gamma a+1) \vec{B}_{T}+(a+1) \vec{B}_{L}-\gamma\left(a+\frac{1}{\gamma+1}\right) \beta \vec{e}_{v} \times \frac{\vec{E}}{c}\right] \times \vec{S}
$$

where $a$ describes the anomalous magnetic moment of the electron given by the higher-order corrections to the eer vertex. In the environment of strong colliding beams, however, the usual perturbation theory cannot be applied. Therefore modified expressions for the anomalous magnetic moment in a medium have been derived [8]. These expressions have been evaluated in the "no scattering" case 
and using the quasi-classical approximation that implies that the change in the momentum due to the strong fields has to be sufficiently slowly. This condition is fulfilled if the Larmor radius of the particle due to the existing magnetic field in the bunches is much larger than the particle wavelength. It has been checked that even in the strong field environment of the ILC such a quasi-classical approximation can be used and the T-BMT equation can be applied to describe the spin precession sufficiently accurate, see also $[6,8,9]$.

\section{Incoherent Background Pairs}

The production of background pairs is strongly dependent on the polarization state of the initial photons involved in the process. These photons are either real (beamstrahlung) or virtual and depend on the constant, crossed bunch electromagnetic fields. The CAIN program contains full polarizations for the real photons, and the Stokes parameter $\left(\xi_{i}\right)$ representation is given in equation 5.510 of the CAIN manual [6]. The polarization of virtual photons depends on the beam electric field $E_{\omega}^{x, y}$ at the point $(x, y)$ where the pair is produced. For gaussian bunches an expression for $E_{\omega}^{x, y}$ is written in terms of a double Fourier transform [10] and is solved by expanding in a Taylor series and using the condition for flat beams $\sigma_{x} \gg \sigma_{y}$

$$
\begin{aligned}
& E_{\omega}^{x}=-\frac{e}{\pi v} \exp \left(-\frac{x^{2}}{2 \sigma_{x}^{2}}\right) \frac{x}{\sigma_{x}^{3}}\left[\exp \left(-\frac{y^{2}}{2 \sigma_{y}^{2}}\right)+y \sqrt{\frac{\pi}{2}} \operatorname{Erf}\left(\frac{y}{\sqrt{2} \sigma_{y}}\right)\right] \\
& E_{\omega}^{y}=\frac{e}{\pi v} \sqrt{\frac{\pi}{2}} \exp \left(-\frac{x^{2}}{2 \sigma_{x}^{2}}\right) \frac{1}{\sigma_{x}} \operatorname{Erf}\left(\frac{y}{\sqrt{2} \sigma_{y}}\right)
\end{aligned}
$$

The Breit-Wheeler cross-section with full polarizations is also required. At present in CAIN this cross-section $\sigma^{\text {circ }}$ is written down only for the product of circular polarizations $\xi_{2} \xi_{2}^{\prime}$ of the two initial photons. The full crosssection $\sigma^{\text {full }}$ is a sum over all polarization states and functions of final electron energy $\epsilon$ and momentum $p$ [11]

$$
\begin{aligned}
\sigma^{\text {circ }} \propto 2\left(1-h_{2}+\frac{2 \epsilon^{2}-1}{2 \epsilon^{2}}\right) \sinh ^{-1} p+\frac{p}{\epsilon}\left(3 h_{2}-1-\frac{1}{\epsilon^{2}}\right) \\
\sigma^{\text {full }} \propto 2\left(1-h_{2}+\frac{2}{\epsilon^{2}}\left(h_{1}+h_{3}\right)-\frac{h_{3}}{\epsilon^{4}}\right) \sinh ^{-1} p \\
\quad+\frac{p}{\epsilon}\left(3 h_{2}-1-h_{1}-\xi_{3} \xi_{3}^{\prime}-\frac{h_{3}}{\epsilon^{2}}\right)
\end{aligned}
$$

where $\quad h_{1}=\xi_{1} \xi_{1}^{\prime} \quad h_{2}=\xi_{2} \xi_{2}^{\prime}$

$$
h_{3}=1+\xi_{3}+\xi_{3}^{\prime}+\xi_{3} \xi_{3}^{\prime}
$$

A numerical investigation of these two cross-sections reveal that the usual peak at low energies (approximately less than $50 \mathrm{MeV}$ ) for the full cross-section is substantially reduced compared to the $\sigma^{\text {circ }}$ prediction.

CAIN was modified with the above expressions and was run for all seven $500 \mathrm{GeV}$ centre of mass collider parameter sets [12]. There was a $10-20 \%$ overall reduction in pairs (figure 1), with no discernible impact on collision luminosity.

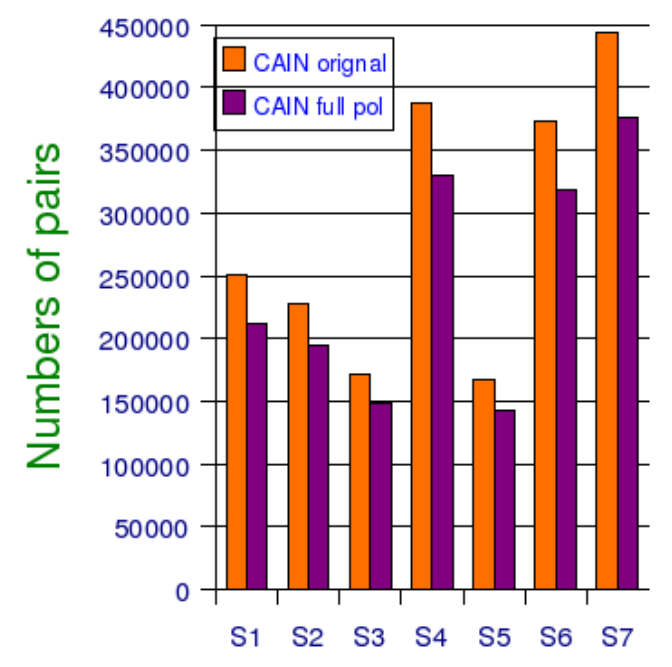

Figure 1: Number of pairs for seven ILC parameter sets.

\section{Coherent Background Pairs}

The coherent production of pairs via the first order interaction between a beamstrahlung photon and the beam field is included already in CAIN. However the second order stimulated Breit-Wheeler process also takes place in the presence of the bunch fields. The cross-section calculation involves solutions of the Dirac equation in an external field (represented by double lines in the Feynman diagram, figure 2).
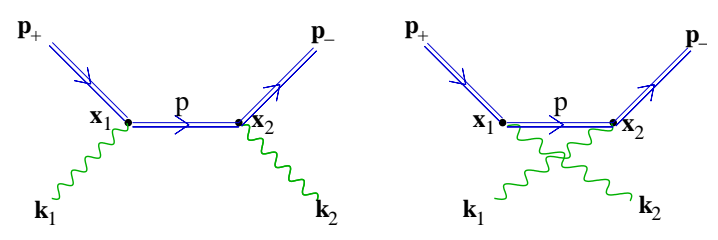

Figure 2: Stimulated Breit-Wheeler Feynman diagrams.

Integrals of products of Airy functions, which are usually associated with constant crossed electromagnetic fields, are introduced into the cross-section. Naively, in comparison to the first order coherent process, the secondorder cross-section is diminished by an order of the fine structure constant. However the bunch field has the effect of allowing the second order cross-section to reach the mass shell. The resulting resonances are rendered finite by inclusion of the electron self-energy and the stimulated Breit-Wheeler cross-section can exceed the first order coherent process. A detailed theoretical and numerical investigation is required to gauge the effect on produced pairs [9].

\section{SPIN TRANSPORT}

The SLICKTRACK [13] Monte Carlo computer code has been used to analyse the spin motion in the ILC damping rings, main linac and beam delivery system. 


\section{Damping Rings}

Previously [7] we have presented results showing that spin motion in the $6 \mathrm{~km}$ DR (OCS) of the ILC, including both spin precession and synchrotron radiation effects in the presence of realistic magnet misalignments $(1 / 3 \mathrm{~mm}$ misalignments and $1 / 3 \mathrm{mrad}$ roll for quadrupole), leads to negligible depolarization both at the design energy of $5.066 \mathrm{GeV}$ and close to a spin-orbit resonance at $4.8 \mathrm{GeV}$.

Updated simulations using SLICKTRACK with the "OCS6" damping ring lattice at $5.0 \mathrm{GeV}$ show that the sum of the mean squares of the angles of tilts of spins away from the direction of the equilibrium polarization (approximately vertical) to be less than $0.1 \mathrm{mrad}^{2}$, even after 8000 turns ( 8 damping times). At $4.8 \mathrm{GeV}$ the sum of the mean squares of the angles was shown to reach approximately $40 \mathrm{mrad}^{2}$ after 8000 turns, which still represents a negligible degree of depolarization. I.e. the ratio of final to initial polarizations in this case is $\cos (\sqrt{40} \mathrm{mrad}) \approx 0.99998$.

These simulations were carried out assuming the narrow energy spread $( \pm 45 \mathrm{keV})$ expected for injected electron bunches. We have also carried our similar simulations for the OCS6 damping ring lattice at $5 \mathrm{GeV}$ assuming an initial energy spread of $\pm 25 \mathrm{MeV}$, much greater than the natural energy spread of the damping ring, as expected for positrons coming from the ILC baseline positron source.

In this case the sum of the mean squares of the spin angles after 8000 turns was found to be approximately $20 \mathrm{mrad}^{2}$ which is once again negligible. It was also shown that in this case, as in the previous simulations, the horizontal projections of the spin vectors of an electron or positron bunch do not fully decohere after 8000 turns, i.e. if the spins are tilted from the vertical at injection then their projections do not fan out uniformly in the horizontal plane during damping, even if the initial energy spread is large. In further work, the effect of the length of the injected positron bunches on this conclusion will be investigated.

\section{Beam Delivery System}

SLICKTRACK has been modified to include a "single pass" mode and then applied to the $2 \mathrm{mrad}$ crossing-angle arm of the older design of the ILC BDS. Realistic misalignments were included as in the damping ring analysis. Simulations showed a total spin precession of approximately 332 degrees and an absolute decrease in the polarization of $0.06 \%$ or less. These figures are consistent with those obtained by Smith et al using the BMAD computer program [5]. Our work will be repeated using updated versions of the BDS lattice consistent with the RDR.

\section{Main Linac}

The SLICKTRACK computer code has also been modified to include acceleration effects and then applied to the ILC main linac. Spin precession of approximately 26 degrees is expected in the Earth-following linac, and SLICKTRACK simulations of the spin motion show the ratio of the final to initial polarizations of particle bunches travelling through the linac is approximately $\cos \left(10^{-4} \mathrm{rad}\right)$.

\section{CONCLUSIONS AND OUTLOOK}

We have studied possible depolarization effects at the ILC at the damping ring, main linac, at the beam delivery system and during the beam-beam interactions for a range of ILC parameters as part of an ongoing rolling study.

-An analysis of depolarization processes during ILC beam-beam interactions was carried out. The current CAIN implementation of depolarization through spin precession has been shown to be valid for ILC energies.

-The polarization dependence of coherent and incoherent pair production at the interaction point has been studied, and these effects are currently being incorporated into CAIN. This work is ongoing, but initial results from CAIN indicate a substantial decrease in low energy incoherent pair production when polarization effects are included.

-The SLICKTRACK software package has been extended to simulate the spin dynamics through the ILC BDS and main linac. All simulations show very small amounts of depolarization, as expected.

- Additional SLICKTRACK simulations of the ILC damping ring lattices support our earlier results showing that the horizontal projections of the spin vectors of the electron and positron bunches injected into the damping rings do not rapidly decohere. It is therefore very important that the vectors be properly aligned prior to injection.

-SLICKTRACK is being extended to include non-linear orbital motion, allowing a detailed study of spin motion in non-linear elements such as sextupoles and wigglers.

\section{REFERENCES}

[1] G. Moortgat-Pick et al., hep-ph/0507011, submitted to Phys.Rep.; www.ippp.dur.ac.uk/ gudrid/power/.

[2] The current ILC Reference Design Report (RDR) can be found on: http://www.linearcollider.org/cms.

[3] D.J. Scott et al., THPMN074, these proceedings.

[4] EUROTeV-Report-2005-024, P. Schmid, A Spin Rotator for the ILC.

[5] ILC-Note-2007-012, J.C. Smith, Depolarization Study in the International Linear Collider.

[6] K. Yokoya, P. Chen, SLAC-PUB-4692, 1988; K. Yokoya, 'User's Manual of CAIN', Version 2.35, April 2003.

[7] EUROTeV-Report-2006-037, G.A. Moortgat-Pick et al., Spin Tracking at the ILC.

[8] V. N. Baier and V. M. Katkov, Phys. Lett. A 280 (2001) 275; V. N. Baier and V. M. Katkov, Phys. Rept. 409 (2005) 261.

[9] G. Moortgat-Pick et al., Cockcroft-07-22 (in preparation).

[10] R. Engel, A. Schiller, V.G. Serbo, Z Phys C, 71 (1996) 651.

[11] V.N. Baier, A.G. Grozin, hep-ph/0209361.

[12] T. Raubenheimer, "Suggested ILC Beam Parameter Range" (2005).

[13] D.P. Barber, et al., Cockcroft-04-01. 